\title{
Hybrid Active Focusing with Adaptive Dispersion for Higher Defect Sensitivity in Guided Wave Inspection of Cylindrical Structures
}

\author{
P. S. Lowe ${ }^{1,2}$, R. Sanderson ${ }^{2}$, N. V. Boulgouris ${ }^{1}$ and T. H. Gan ${ }^{2}$ \\ ${ }^{1}$ Brunel University, Kingston Lane, Uxbridge, Middlesex UB8 3PH, UK \\ ${ }^{2}$ TWI Ltd, Granta Park, Great Abington, Cambridge, CB21 6AL, UK (e-mail: \\ eepgpsw@brunel.ac.uk and shehan.lowe@affiliate.twi.co.uk)
}

\begin{abstract}
Ultrasonic Guided Wave inspection is widely used for scanning prismatic structures such as pipes for metal loss. Recent research has investigated focusing the sound energy into predetermined regions of a pipe in order to enhance the defect sensitivity. This paper presents an active focusing technique which is based on a combination of numerical simulation and time reversal concept. The proposed technique is empirically validated using a 3D laser vibrometry measurement of the focal spot. The defect sensitivity of the proposed technique is compared with conventional active focusing, time reversal focusing and synthetic focusing through an empirically validated finite element parametric study. Based on the results, the proposed technique achieves approximately $10 \mathrm{~dB}$ improvement of signal-to-coherent-noise ratio compared to the conventional active focusing and time reversal focusing. It is also demonstrated that the proposed technique to have an amplitude gain of around $5 \mathrm{~dB}$ over synthetic focusing for defects $<0.5 \lambda_{s}$. The proposed technique is shown to have the potential to improve the reliably detectable flaw size in guided wave inspection from $9 \%$ to less than $1 \%$ cross sectional area loss.
\end{abstract}

Keywords : ultrasonic guided waves, active focusing, defect sensitivity, finite element analysis, pipe inspection

\section{Introduction}

Pipelines are used extensively in the industry for large-scale distribution of fluids such as crude oil and water. As pipelines age, corrosion flaws can develop and it is therefore important to find techniques to inspect them efficiently. Defective pipelines can lead to fatalities, property damage, litigation and damage to the environment. Pipelines are also often inaccessible and insulated. Because of this, Ultrasonic Guided Wave (UGW) inspection has attracted a great deal of interest as a non-destructive testing technique in the past two decades [1]. The UGW based techniques offer the advantage of full volumetric inspection of tens of metres of pipeline from a single test location. However, the behaviour can be complex and must be understood in order to apply the technique effectively. Initially, the UGW was developed as a low resolution rapid scanning technique to find relatively large defects. However, there is a demand to expand knowledge on the UGW inspection to allow more complex structures to be inspected and smaller defects to be detected i.e. pitting corrosion. The Cross Sectional Area (CSA) loss of pitting corrosion is smaller than that which can be detected due to the low resolution of the UGW. The UGW focusing is one of the techniques that can be used to detect smaller defects (defect circumferential extent $\leq 1.5 \lambda$ ). 


\section{UGWs in cylindrical structures}

Early research into the use of UGW to inspect cylindrical structures was performed by Silk and Bainton [2]. They investigated the use of UGW to inspect small diameter tubes and discussed the equivalence of the waves in tubes to Lamb waves in plates. Nomenclature for identifying UGW modes in cylindrical structures is essential as there are an infinite number of UGW modes possible. The nomenclature used throughout this paper is that which was popularized by Silk and Bainton [2] and initially suggested by Meitzler [3]. According to this nomenclature, the wave modes in cylindrical structures can be represented in the following format, $X(n, M)$. Where, $X$ denotes whether the wave modes are Torsional, Longitudinal or Flexural, $n$ is the positive number of cyclic variations of displacement around the circumference and $M$ is an index which also relates to the level of complexity of vibration within the pipe wall.

A general solution of harmonic wave propagation of infinitely elongated hollow cylinders was presented by Gazis in 1959 [4]. His study has become one of the foundations in the understanding of UGW behaviour in cylindrical structures. There are a higher number of possible axisymmetric modes and an infinite number of nonaxisymmetric (flexural) modes with propagation behaviour corresponding to their axisymmetric mode [5]. For example, the axisymmetric wave mode with torsional displacement characteristics, $\mathrm{T}(0,1)$, has a so-called family of flexural wave modes with torsional displacement characteristics and velocities that tend to the $\mathrm{T}(0,1)$ velocity: $\mathrm{F}(i, 2), i=\{1,2 .$.$\} .$

The dispersion curve diagram illustrates the velocity of the wave mode in relation to the frequency (or wavenumber) with separate curves for each of the existing modes in a frequency region. Sample dispersion curves for an 8inch Schedule 40 (219.1mm outer diameter, $8.18 \mathrm{~mm}$ wall thickness) steel pipe are presented in Fig. 1 [6]. The three main axisymmetric wave modes $(\mathrm{L}(0,1), \mathrm{T}(0,1)$ and $\mathrm{L}(0,2))$ are shown in black lines and their respective families of flexural wave modes are shown in blue lines. The dispersion is the signal spreading out or distorting throughout time and space while it propagates through a structure. The dispersion can limit inspection resolution because of losses in signal amplitude relative to the noise level. Also, the level of dispersion in a particular wave mode can affect data interpretation [7, 8]. As can be seen in Fig. 1, The $\mathrm{T}(0,1)$ wave mode is non-dispersive at all frequencies and the $\mathrm{L}(0,2)$ wave mode exhibits very little dispersion at higher frequencies (approximately above $30 \mathrm{kHz}$ in this example). For this reason, these are the modes that are most commonly used for UGW inspection of hollow cylinders.

In the present study, the $\mathrm{T}(0,1)$ wave mode and its family of flexural modes $(\mathrm{F}(i, 2), i=\{1,2 .\}$.$) are used. Fig. 2, illustrates the displacement characteristics of the$ $\mathrm{T}(0,1)$ wave mode and its first flexural wave mode, $\mathrm{F}(1,2)$ in a short section of 8inch Schedule 40 steel pipe (outer diameter $-219.1 \mathrm{~mm}$ and wall thickness $-8.18 \mathrm{~mm}$ ). The images show, the standing waves calculated using a Finite Element (FE) procedure for dispersion curve calculation developed by Sanderson [9]. The standing waves are calculated using boundary conditions to ensure they have identical characteristics to propagating waves [9]. It can be seen that the $\mathrm{T}(0,1)$ mode is dominated by circumferential displacements [10]. 


\section{Comparison of focusing techniques}

Focusing is a relatively new technique where the UGW energy is concentrated at a particular position both along the axis and around the circumference of a pipe. A number of focusing techniques have been presented in the literature: time reversal focusing [11], active focusing [12-16] and synthetic focusing [17]. Each of these techniques has its own benefits and limitations which are discussed here.

Prada and Fink [18] carried out early research on the time reversal concept. The conventional time reversal process takes advantage of several aspects of piezoelectric transducers such as transmit and receive reciprocity, linearity and capability of instantaneous measurement of the waveforms. A so-called Time Reversal Mirror (TRM) is used. Pressure wave forms, $p\left(r_{i}, t\right)$ are recorded at each transducer location $r_{i}$ over time, $t$ from a potential defect. This data is stored over a selected time period, $T$. The pressure wave is then retransmitted with the data chronologically reversed which is equivalent to the transmission of $p\left(r_{i}, T-t\right)$ [19]. When applied to guided waves, the time reversal focusing uses the sound energy that was reflected back to the transmitted position after the incident pulse has interacted with a non-axisymmetric feature or defect $[11,20,21]$. The received signal is then time reversed and retransmitted, and this result in the signal re-converging at the defect location as a superposition of the individual wave modes reflected from the feature [11]. The time reversal focusing is useful for testing components of irregular geometries or anisotropic materials as the unpredictable distortions and dispersion of the signal will be automatically corrected by the time reversal function due to the elastic properties of UGWs. However, the time reversal focusing relies on the signals from conventional UGW inspection and for smaller defects (pitting corrosion) the reflected signal amplitude will be close to, or even below the noise level. Therefore, for signal amplitudes close to the noise level, the performance of the time reversal focusing will be compromised.

Excitation of non-axisymmetric modes by applied non-axisymmetric loading was studied by Shin and Rose [22]. Based on this study, the active focusing concepts were developed [12-16, 23]. Angular profile tuning of a transducer was studied by Li and Rose [24] to detect defects at a focal spot where the UGW energy was concentrated at a given axial location as a function of circumferential position. The active focusing technique calculates the input parameters required for focusing at a preselected location prior to testing [13-16, 23]. The calculations use the phase velocities of the axisymmetric wave mode and non-axisymmetric modes to determine time delays and amplitude scaling factors to apply to the array with the aim of making all the wave modes arrive at the same location at once resulting in the focal spot [23]. However, in this technique, dispersion of the waves is not taken into account. Therefore, the active focusing tends to operate well at higher frequencies and/or in larger diameter pipes where there are a large number of relatively non-dispersive flexural wave modes are in existence. But not at low frequencies (or in smaller diameter pipes) where there are fewer flexural wave modes and dispersion is more significant. Furthermore, in the UGW inspection, the capability of focusing at lower frequency $(20-100 \mathrm{kHz})$ is significant. Also the active focusing techniques need extensive hardware, separate signals generators and amplifications for each transmitting point.

On the other hand, the synthetic focusing technique is a post processing approach where the results can be obtained from conventional UGW inspection data where, different pairs of transducers are used to transmit and receive signals. Therefore, the data acquisition time required to inspect the whole volume of the pipe will be considerably shorter compared with the other techniques [17, 25]. However, the time 
saved at the data acquisition step is partially counteracted by increased computation time required for post processing the received signals. With the evolution of technology, this can be compensated as the synthetic focusing method is ideally suited for parallel processing algorithms. However, there is a reduction in the SNR of the synthetic focusing compared with the active focusing techniques [26].

The proposed hybrid focusing technique is based on a combination of numerical simulation with active focusing and time reversal concept which is referred to as Hybrid Active Focusing (HAF) from hereon in. The proposed technique is designed for application in a typical UGW frequency range (20-100 kHz). The HAF compensates for dispersion as it adopts the time reversal concept due to the elastic properties of UGWs [18]. Therefore the HAF technique can be used at any frequency to enhance the UGW resolution to detect defects. The FE results of the performance of the proposed technique have been empirically validated using modified commercially available UGW hardware and 3D Laser Doppler Vibrometry (3D-LDV) [27]. The proposed technique is compared with active focusing [23] and time reversal focusing [11] and the comparison is based on Signal-to-Coherent-Noise-Ratio (SCNR) for a range of defects (circumferential extent $\leq 1.5 \lambda$ ). Furthermore the proposed technique is compared to the synthetic focusing based on the input signal to defect amplitude presented by Davies and Cawley [25].

The paper is organized as follows: In Section II, the theory behind the proposed focusing technique is presented. Then, the FE analysis and empirical validation of the performance of the proposed technique against unfocused UGW is presented in Sections III and IV respectively. Section V presents, the performance of the HAF compared to the active focusing, time reversal focusing and the synthetic focusing against a range of circumferential crack-like defects.

\section{Proposed focusing theory}

The proposed focusing technique is a hybrid active focusing technique which combines FE modelling with time reversal concept $[11,19]$. With the continual increase in computational power and the introduction of semi analytical finite element methods [28, 29], it is becoming possible to obtain wave propagation predictions in realistically sized structures in a few seconds. This makes the computation of the input signals required to achieve focusing possible as part of a practical inspection procedure.

The HAF technique proposed in this paper can be explained as follows:

Step I - focusing input signals are calculated by exciting a non-axisymmetric pressure wave at a point at the desired focal location in a FE model. In this example, a Hann-windowed pulse has been used and the excitation was applied in the circumferential direction as follows:

$$
U_{\theta}(t)=\frac{1}{2} \sin (2 \pi f t)\left[1-\cos \left(\frac{2 \pi f t}{n}\right)\right]
$$

where, $t$ is time, $f$ is the central frequency and $n$ is the number of cycles.

Step II - record the transmitted input signals in the FE model at the locations where the transducers will be placed in the experiment. A typical signal received at a single transducer at a distance from the applied non-axisymmetric excitation is shown in Fig. 3-a.

Step III - Then, a temporal gate is applied to the received signals as illustrated by the dashed red vertical lines in Fig. 3-b. The limits of the temporal gate are 
dependent on a compromise between excitation time and refinement gained from the inclusion of higher order (but most likely lower amplitude) flexural wave modes. The lower limit of the temporal gate is the Time-of-Fight (ToF) of the excited axisymmetric mode and the upper limit of the gate is related to the ToF of the highest desired flexural mode in the selected frequency. The signal can also be truncated based on using an assumed amplitude ratio as a cut-off.

Step IV - the set of recorded signals are time reversed (as shown in Fig.3-c) and applied as excitations to the actual transducers.

Step V - finally, the signals are recorded at the transducers as in any standard inspection (sum the signals around the circumference and account for directionality using a number of rings of transducers) and the received level of the axisymmetric wave mode is assessed.

\section{Finite element analysis}

FE analysis has been performed to study the performance of the HAF technique compared with unfocused axisymmetric excitation. A 3D model was built using ABAQUS/EXPLICIT version 6.13 [30]. The geometry modelled was an 8inch Schedule 40 steel pipe (outer diameter $-219.1 \mathrm{~mm}$ and wall thickness $-8.18 \mathrm{~mm}$ ) with an axial length of $2.5 \mathrm{~m}$. The assumed material properties for steel were as follows: Density $(\rho)=$ $7830 \mathrm{~kg} / \mathrm{m}^{3}$, Young's modulus $(\mathrm{E})=207 \mathrm{GPa}$ and Poisson's ratio $(v)=0.3$. Linear eight node brick elements with reduced integration (ABAQUS element type C3D8R) were used to achieve efficient computation time and the mesh refinement was such that there were at least eight elements for the smallest possible wave length in the main lobe of the frequency bandwidth. This level of mesh refinement was validated in previous studies [31- 33]. Excitation and reception were performed using 24 equally spaced circumferential points to match with the number of transducers around the circumference in the tooling used in the empirical study. The use of points in the current FE method is considered to be sufficient for the present study since the wave length of the $\mathrm{T}(0,1)$ is larger than the size of the transducers elements [34] used in the empirical study. The simulation of the transducers as a point source for guided wave applications have also been previously validated by a number of authors [10, 29, 35]. In the next section, we present the initial model used to calculate the focusing parameters required for the HAF technique as well as the models used to study the performance of the HAF compared with unfocused axisymmetric excitation.

\section{Calculating focusing inputs for HAF technique}

As explained in Section II, a point excitation was simulated in an FE model in order to generate the focusing parameters required for the HAF technique. The excitation used for this study was a 10-cycle $50 \mathrm{kHz}$ (length of the shear wavelength $\left(\lambda_{s}\right)=64 \mathrm{~mm}$ ) Hann-windowed pulse with loading in the circumferential direction at a propagation distance of $1.5 \mathrm{~m}$ from the reception points. The received displacements are therefore multimodal signals, which contain a mixture of the axisymmetric mode and its corresponding flexural modes. In this case, due to the circumferential loading, the signal will be dominated by $\mathrm{T}(0,1)$ and its family of corresponding flexural modes, $\mathrm{F}(i, 2), i=$ $\{1,2, \ldots\}$.

The layout of the FE model is illustrated in Fig. 4. To avoid reflections from the free ends of the pipe, absorbing boundary conditions were used as shown in Fig. 4. This avoids the need for simulation of a longer pipe and therefore reduces the required 
computation time. The absorbing boundary was achieved by use of the 'infinite element' ((ABAQUS element type CIN3D8) [30]) in Abaqus. This consists of elements defined over semi-infinite domains with suitably chosen decay functions [36]. The input signals were then processed following the procedure described in Section II and illustrated in Fig. 3.

\section{Performance of the HAF compared with standard unfocussed axisymmetric excitation}

Two FE models were generated to study the performance of the HAF compared with the standard unfocused excitation. The two cases studied are summarised as follows:

Case 1 (unfocused axisymmetric excitation): The input tone-burst was a 10-cycle 50 $\mathrm{kHz}$ Hann windowed pulse excited at 24 transmitting points spaced evenly around the circumference.

Case 2 (HAF): The time reversed displacement inputs produced by the model described in Section III-A, were used. Separate input signals were applied to each of the 24 evenly spaced points around the circumference corresponding to the same circumferential position from which they were collected.

Fig. 5 shows the results of the two cases. Figs. 5-a \& b show the contour plots of the displacement magnitude at the time of focusing $1.5 \mathrm{~m}$ from the transmission location. Fig. 5-c shows a polar plot of the normalized amplitude (normalized to the max amplitude of the results) $1.5 \mathrm{~m}$ from the transmission location. The amplitude gain can be observed between the focused case with a well-defined peak at the $0^{\circ}$ location and the unfocused case which is uniform around the circumference as expected.

\section{Experimental validation}

In order to validate the results based on the FE analysis in Section III, laboratory experiments were performed on an 8inch Schedule 40 steel pipe (outer diameter $219.1 \mathrm{~mm}$ and wall thickness $-8.18 \mathrm{~mm}$ ). A 3D-LDV was used to measure the vibration of the pipe surface and obtain measured amplitudes and time of arrival information. The 3D-LDV is equipped with three laser sensor heads in order to detect the surface vibration caused by UGW propagation through the structure. The laser beam from the 3D-LDV was focused at the surface of interest, and used to extract the velocity amplitude from the "Doppler shift" of the laser beam frequency arising from the displacement of the surface $[27,37]$.

\section{Experimental setup}

The overall experimental setup is illustrated in Fig. 6-a, and commercially available hardware (Teletest ${ }^{\circledR}$ Focus+ [34]) was used for the experiment. A modified version of the software was used to excite the non-standard signals required to achieve focusing (see Section III-A). The hardware was also modified from the conventional set up so that a single ring of 24 evenly spaced individually addressable piezoelectric transducers can be used for excitation instead of three rings of transducers grouped into octants. As per the standard UGW testing procedure, the piezoelectric transducers were dry-coupled to the specimen. The pipe was resting on two rubber pipe rollers throughout the experiment to avoid interference and loss of energy into the surroundings and to allow 
the pipe to be easily rotated.

In order to capture an appropriate region of the waveform, the minimum length to scan was calculated as follows,

$$
L=n\left(\frac{V_{g r}}{f}\right),
$$

where, $n$ is the number of cycles, $V g r$ is the highest possible group velocity at the selected frequency, $f$ and therefore, the length of line scan required can be calculated as equal to, or greater than $L$. The highest possible group velocity was $3800 \mathrm{~m} / \mathrm{s}$, and so the $3 \mathrm{D}-\mathrm{LDV}$ was therefore used to scan along a $0.95 \mathrm{~m}$ line on the pipe surface.

Two types of data collections were carried out to measure the performance of the focusing technique both qualitatively and quantitatively:

Data collection 1: to extract qualitative data, an area scan of the pipe surface was collected over an axial length of $0.95 \mathrm{~m}$ and covering $90^{\circ}$ of the pipe circumference as illustrated in Fig. 6-b.

Data collection 2: to extract quantitative data, a line scan along an axial length of $0.95 \mathrm{~m}$ was collected. The pipe was rotated 24 times at $15^{\circ}$ intervals using pipe rollers to obtain 24 individual line scans at different circumferential positions as illustrated in Fig. 6-c.

\section{Experimental results}

The measured surface velocity of the pipe at a fixed point in time collected using the area scan is shown in Fig. 7 for both the unfocused axisymmetric excitation (Fig. 7-a) and focusing using the HAF (Fig. 7-b). Since the velocity is the rate of change of displacement with time, it is possible to use either when assessing the characteristics of the waveforms. There is a good qualitative agreement between the patterns and those predicted by the model (Fig. 5). Fig. 7-c, shows the normalized amplitude obtained from the line scans measured at different circumferential positions $1.5 \mathrm{~m}$ away from the excitation location directly compared with the FE predictions.

The expected shape of the focal spot as measured by the 3D-LDV agrees well with that predicted using FE analysis in Section III-B. There is also good agreement between the measured amplitudes of the focal spot at different circumferential locations and the FE predictions at those locations. This gives confidence in the validity of both the FE model and the practical application of proposed HAF technique.

\section{Parametric study}

An empirically validated FE model (see Sections III and IV) has been used to further investigate the performance of the HAF. A parametric study has been performed to investigate defect sensitivity of the HAF compared to the active focusing [23], time reversal focusing [11] and synthetic focusing [25] techniques. A set of fully penetrating, part-circumferential crack-like defects were considered in an 8inch Schedule 40 steel pipe (outer diameter $-219.1 \mathrm{~mm}$ and wall thickness $-8.18 \mathrm{~mm}$ ). The dimensions of the defects modelled can be found in Table 1 and Fig. 8 illustrates the geometry of the defects modelled.

The HAF is an active focusing technique which is used to enhance the resolution at a particular location. Therefore, the performance of the HAF is compared to the active focusing [23] and also compared to the time reversal focusing [11] as it adopts the time reversal concept. This comparison is based on SCNR for a range of defects 
with circumferential extent of $3.5^{\circ}, 7^{\circ}, 15^{\circ}, 30^{\circ}$ and $60^{\circ}\left(0.08 \lambda_{s}, 0.16 \lambda_{s}, 0.32 \lambda_{s}\right.$, $0.72 \lambda_{s}$ and $1.44 \lambda_{s}$ respectively). Furthermore the proposed technique is compared to the synthetic focusing based on the input signal to defect amplitude ratio published by Davies and Cawley [25].

In each case (Table 1), the crack-like defect was placed exactly at the desired focal spot location $\left(1.5 \mathrm{~m}\right.$ from the excitation and centered at $\left.0^{\circ}\right)$. In a real application, the defect location may be an unknown. If this is the case, the excitation location of the focusing parameters can be easily rotated to enable a sweep of the circumference to be obtained. However, in order to cover different axial locations, additional models to calculate the focusing inputs would need to be performed. This could be performed relatively rapidly if a semi analytical model is used. However, in such a case it may be faster to use a synthetic focusing technique to map the condition of the pipe [38].

In a standard UGW inspection, where an unfocused axisymmetric excitation is used, generally the reliably detectable defect size is commonly accepted to be 9\% CSA loss [11]. Therefore, depending on the test conditions, defects smaller than this may be below the noise level and therefore remain undetected. The noise is made up of a mixture of randomized noise such as that arising from electrical interference or coherent noise which is the presence of unwanted wave modes appearing as the results of events such as reflection from the structural features i.e. pipe supports, transmission, mode conversion and dispersion [39]. The level of random noise can be mitigated by taking an average of a number of repeat tests. Therefore, it is commonly the coherent noise that contributes more significantly to the noise level. It is also the coherent noise that is predicted by the FE models, as the random noise cannot be predicted using FE models. In this study the coherent noise is caused by the propagation of unwanted modes and the dispersive behaviour (refer Fig. 1) of the higher order modes. In this FE study, the detectability has therefore been assessed using SCNR. The calculation of SCNR is graphically illustrated in Fig. 9 using the time-domain response from Defect ID: D-3 and SCNR is calculated as follows:

$$
S C N R=20 \log _{10}\left(\frac{A_{D}}{A_{N}}\right)
$$

where, $A_{D}$ is the max amplitude of the defect signal and $A_{N}$ is the max amplitude of the nearby coherent noise.

The results of the parametric study to compare the performance of the HAF with the active focusing and the time reversal focusing are illustrated in Fig. 10 and summarised in Table 2. It can be seen that the active focusing technique has a higher defect sensitivity compared with the time reversal focusing technique particularly for smaller defects and that the HAF technique is a significant improvement upon that.

Furthermore, the HAF is compared to unfocused axisymmetric excitation and the synthetic focusing based on the input signal to defect amplitude presented by Davies and Cawley [25]. The input and defects amplitudes are calculated using eq-(4). The reflection of the $\mathrm{T}(0,1)$ wave mode from cracks in pipes has been thoroughly investigated by Demma et al. [40]. The reflection ratio of the $\mathrm{T}(0,1)$ wave mode from through-thickness cracks was found to be equal to the crack circumferential extent as a fraction of the pipe circumference. The results are illustrated in Fig. 11 and shows that the HAF has around $5 \mathrm{~dB}$ amplitude gain over the synthetic focusing. Based on a $6 \mathrm{~dB}$ criterion for detection [34], the HAF technique improves the reliably detectable flaw size from $9 \%$ to less than $1 \%$ CSA loss. Further discussion on the results of the parametric study can be found in Section VI. 


\section{Further analysis}

As seen in the previous section, the HAF technique achieves at least $10 \mathrm{~dB}$ SCNR improvement over the active focusing and the time reversal focusing. Furthermore, the $0.08 \lambda_{s}$ circumferential extent defect could not be detected using the active focusing and the time reversal focusing due to the lack of sensitivity, but the proposed technique was able to detect the $0.08 \lambda_{s}$ circumferential extent defect with 13dB SCNR. Furthermore, the synthetic focusing and the HAF techniques perform similarly at defects over $1 \lambda_{s}$ circ. extent. However, the proposed method achieves around $5 \mathrm{~dB}$ amplitude gain when the circ. extent of the defect is less than $0.5 \lambda_{s}$. The HAF performs well for smaller defects (circ. extent $0.5 \lambda_{s}$ ). This is because the HAF technique uses a controlled nonaxisymmetric pulse (contains all the non-axisymmetric modes in the frequency bandwidth) with high defect sensitivity. This does not depend on experimentally gathered data unlike the time reversal focusing and synthetic focusing. However, the results examined to the date are based a limited number of cases and further work involving a larger modelling and experimental study would be beneficial in establishing the potential of the HAF method to detect smaller defects.

The time reversal focusing depends on data gained from an axisymmetric excitation (received non-axisymmetric wave modes depend on the size of the defect). It is therefore difficult to detect defects using the time reversal focusing, if the defects are not detectable by unfocused axisymmetric excitation in the first place.

Furthermore, the HAF technique compensates for dispersion by using time reversal concept, whereas the active focusing technique neglects dispersive behaviour. Therefore, the HAF technique has the advantage of operating well at low frequencies or in small diameter pipes. Yet, the HAF technique requires a different set of input signals for every axial position. Therefore, there are many tests required to cover a large area which could be time consuming. However, analytical models for wave propagation in pipes can be used to provide fast calculations of the input signals required [29] which can be incorporated into the inspection process. As the HAF is an active focusing technique, it is likely to have a higher SNR compared to synthetic focusing techniques [26].

However, the performance of the HAF technique in the field trials can be compromised if the size of the pipe deviate largly from the modelled pipe. Therefore, further studies need to take place on-site to quantify this effect. Furthermore, the stability of the HAF technique needs to be experimentally studied on varying defect profiles, operating temperatures and focusing beyond known featuers i.e welds and branches.

\section{Conclusion}

A high sensitivity focusing technique has been presented that is useful in low frequency UGW inspection of cylindrical structures which compensates for dispersion and operates in low frequency. The proposed technique, termed hybrid active focusing is based on a combination of numerical simulation with the active focusing and time reversal concept. The defect detection performance of the new technique was compared with the active focusing, the time reversal focusing and the synthetic focusing using an empirically validated FE method. The FE analysis was validated against 3D laser vibrometry measurements of the focal spot and UGW unfocused excitation.

It was shown that the proposed technique is expected to have a $10 \mathrm{~dB}$ SCNR improvement over the active focusing and the time reversal focusing. Furthermore, 
based on the comparison of the HAF and the synthetic focusing it was shown that the both methods perform similarly at defects $>1 \lambda_{s}$ circ. extent. However, The HAF expected to have an amplitude gain of $5 \mathrm{~dB}$ over the synthetic focusing for defects smaller than $0.5 \lambda_{s}$. These results exhibit the potential of using the HAF for refined inspection of pipelines.

\section{Acknowledgement}

The authors gratefully acknowledge TWI Ltd and the Center for Electronic System Research (CESR) of Brunel University for providing the funding that made this study possible. The authors are also grateful to Prof. P. Cawley of Imperial College, London Prof. W. Balachandran of Brunel University, London and Prof. P. Mudge of Plant Integrity Ltd., for their constructive inputs and many useful discussions.

\section{References}

[1] J. L. Rose, "Standing on the shoulders of giants: An example of guided wave inspection," Materials Evaluation, vol. 60, pp. 53-59, 2002.

[2] M. G. Silk and K. F. Bainton, "The propagation in metal tubing of ultrasonic wave modes equivalent to lamb waves," Ultrasonics, pp. 11-19, 1979.

[3] A. H. Meitzler, "Mode coupling occurring in the propagation of elastic pulses in wires," Journal of Acoustical Society of America, vol. 33, no. 4, pp. 435-445, 1961.

[4] D. C. Gazis, "Three-dimensional investigation of the propagation of waves in hollow circular cylinders. I. Analytical foundation," Journal of Acoustical Society of America, vol. 31, no. 5, pp. 568-573, 1959.

[5] D. C. Gazis, "Three-dimensional investigation of the propagation of waves in hollow circular cylinders. II. Numerical results," Journal of Acoustical Society of America, vol. 31, p. 573-578, 1959.

[6] R. Sanderson, "A closed form solution method for rapid calculation of guided wave dispersion curves for pipes," Wave Motion, vol. 53, pp. 40-50, 2014.

[7] P. D. Wilcox, "A rapid signal processing technique to remove the effect of dispersion from guided wave signals," IEEE Transaction on Ultrasonics, Ferroelectrics and Frequency Control, vol. 50, no. 4, pp. 419-427, 2003.

[8] P. D. Wilcox, M. J. S. Lowe and P. Cawley, "Mode and trasnducer selection of long range lamb wave inspection," Journal of Intelligent Material Systems and Structures, vol. 12, pp. 553-565, 2001.

[9] R. Sanderson and S.D. Smith, "The application of finite element modelling to guided wave testing system," Review of Quantitative Nondestructive Evaluation, vol. 22, p. 256.263, 2003.

[10] P. Catton, "Long range ultrasonic guided waves for the quantitative inspection of pipelines," PhD Thesis, pp. 10-20, 2009.

[11] C. Ennaceur, P. Mudge, B. Bridge, M. Kayous and T.H. Gan, "Application of the time reversal technique to the focusing of long-range ultrasound in pipelines," Insight, vol. 49, no. 4, pp. 217-223, 2007.

[12] J. Li and J. L. Rose, "Excitation and propagation of non-axisymmetric guided waves in a hollow cylinder," Journal of Acoustical Society of America, vol. 109, no. 2, p. 457-464, 2001. 
[13] Z. Sun, L. Zhang and J. L. Rose, "Flexural torsional guided wave mechanics and focusing in pipe," Journal of Pressure Vessel Technology, vol. 127, p. 471-478, 2005.

[14] J. Li and J. L. Rose, "Natural beam focusing of non-axisymmetric guided waves in large-diameter pipes.," Ultrasonics, vol. 44, p. 35-45, 2006.

[15] T. Hayashi, K. Kawashima, Z. Sun and J. L. Rose, "Analysis of flexural mode focusing by a semianalytical finite element method," Journal of Acoustical Society of America, vol. 113, no. 3, p. 1241-1248, 2003.

[16] J. Mu, J. Zhang and J. L. Rose, "Defect circumferential sizing by using long range ultrasonic guided wave focusing techniques in pipe," Nondestructive Testing and Evaluation,, vol. 22, no. 4, p. 239-253, 2007.

[17] J. O. Davies, "Inspection of pipes using low frequency focused guided waves," Imperial College London - PhD Thesis, 2008.

[18] C. Prada \& M. Fink , "Eigenmodes of the time reversal operator: A solution to selective focusing in multiple-target media," Wave Motion, vol. 20, pp. 151-163, 1994.

[19] M. Fink, "Time reversal of ultrasonic fields-part I: Basic principles," IEEE Transactions on Ultrasonics, Ferroelectrics and Frequency Control, vol. 39, no. 5, pp. 555-566, 1992.

[20] Y. Nagashima, M. Endon and M. Koike, "Signal transmission technique of dispersive guided waves for long-range inspection," in 4th International Conference on NDE in Relation to Structural Integrity for Nuclear and Pressurised Components, 2004.

[21] F. Deng, C. He and B. Wu, "Time reversal method for pipe inspection with guided waves," in Thomson, D. O., \& Chimenti, D. E. (eds), Review of Progress in Quantitative Non-Destructive Evaluation, vol. 975. Golden, CO: AIP Conference Proceedings, Golden, Colorado, 2007.

[22] H. J. Shin and J. L. Rose, "Guided waves by axisymmetric and non-axisymmetric surface loading on hollow cylinders," Ultrasonics, vol. 37, p. 355-363, 1999.

[23] J. L. Rose and P. J. Mudge, "Flexural mode focusing in a pipe," in 8th ECNDT, Barcelona, 2002.

[24] J. Li and J. L. Rose, "Angular-profile tuning of guided waves in hollow cylinders using a circumferential phased array," IEEE Transactions on Ultrasonics, Ferroelectrics, and Frequency Control, vol. 49, no. 12, pp. 1720-1729, 2002.

[25] J. Davies and P. Cawley, "The Application of Synthetic Focusing for Imaging Crack-Like Defects in Pipelines Using Guided Waves," IEEE Transactions on Ultrasonics, Ferroelectrics, and Frequency Control, vol. 56, no. 4, pp. 759-771, 2009.

[26] C. Holmes, B. W. Drinkwater and P. D. Wilcox, "Post-processing of the full matrix of ultrasonic transmit-receive array data for non-destructive evaluation," $N D T \& E$ International, vol. 38, no. 8, p. 701-711, 2005.

[27] R. Behrendt, S. Heuer and H. Steger, "3D Scanning Laser Vibrometry,” Polytec,, Hamburg, Germany, 2005.

[28] L. Gavric, "Computation of propagative waves in free rail using finite element technique," Journal of Sound and Vibration, vol. 185, pp. 531-543, 1995.

[29] R. M. Sanderson and P. P. Catton, "An analytical model for guided wave inspection optimization for prismatic structures of any cross section," IEEE 
Transactions on Ultrasonics, Ferroelectrics and Frequency Control, vol. 58, no. 5, pp. 1016-1026, 2011.

[30] ABAQUS, “ABAQUS Version 6.13 user's maual,” Simulia, 2013.

[31] M. J. S. Lowe, D. N. Alleyne and P. Cawley, "The reflection of guided waves from circumferential notches in the pipe," Journal of Applied Mechanics, vol. 65, no. 3, pp. 635-641, 1998.

[32] P. S. Lowe, S. Fateri, R. Sanderson and N. V. Boulgouris, "Finite element modelling of the interaction of ultrasonic guided waves with coupled piezoelectric transducers," Insight, vol. 56, no. 9, pp. 505-509, 2014.

[33] S. Fateri, P. S. Lowe, B. Engineer, and N. V. Boulgouris, "Investigation of Ultrasonic Guided Waves Interacting with Piezoelectric Transducers," IEEE Sensors Journal, p. DOI 10.1109/JSEN.2015.2414874, 2015.

[34] P. Mudge, "Field application of the Teletest ${ }^{\circledR}$ long-range ultrasonic testing technique," 2001.

[35] D. Alleyne, "The nondestructive testing for plates using ultrasonic lamb waves," Imperial College -Ph.D Thesis, London, 1991.

[36] J. Lysmer \& R. L. Kuhlemeyer, "Finite Dynamic Model For Infinite Media," Journal of the Engineering Mechanics Division, vol. 95, no. 4, pp. 859-878, 1969.

[37] D. E. Oliver, "Tutorial: 3D scanning vibrometry for structural dynamics measurements," Polytec, Hamburg, Germany, 2007.

[38] T. Hayashi and M. Murase, "Defect imaging with guided waves in a pipe," Journal of Acoustical Society of America, vol. 117, no. 4, p. 2134-2140, 2005.

[39] P. Cawley, "Practical long range guided wave inspection - managing complexity," in Second Middle East Nondestructive Testing Conference and Exhibition, Saudi Arabia, 2003.

[40] A. Demma, P. Cawley, M. Lowe, and A. G Roosenbrand, "The reflection of fundamental torsional mode from cracks and notches in pipes," Journal of Acoustical Society of America, vol. 114, no. 3, p. 611-625, 2003. 
Table 1: Description of the crack-like defects considered in the study

\begin{tabular}{lllll}
\hline \hline Defect ID & Depth, mm & Circ. extent, $\mathrm{mm}$ & $\begin{array}{l}\text { Axial length, } \\
\mathrm{mm}\end{array}$ & Circ. Extent $\lambda_{s}$ \\
\hline D-1 & 8.18 & $6.85\left(3.75^{\circ}\right)$ & 1 & 0.08 \\
D-2 & 8.18 & $13.7\left(7.5^{\circ}\right)$ & 1 & 0.16 \\
D-3 & 8.18 & $27.4\left(15^{\circ}\right)$ & 1 & 0.36 \\
D-4 & 8.18 & $54.75\left(30^{\circ}\right)$ & 1 & 0.72 \\
D-5 & 8.18 & $109.5\left(60^{\circ}\right)$ & 1 & 1.44 \\
D-5 & 8.18 & $172.1\left(90^{\circ}\right)$ & 1 & 2.15 \\
\hline \hline
\end{tabular}


Table 2: Comparison between the proposed method and previous methods

\begin{tabular}{llll}
\hline Circ. Extent $\lambda_{s}$ & $\begin{array}{l}\text { Time Reversal } \\
\text { Focusing [11] } \\
\text { SCNR, dB }\end{array}$ & $\begin{array}{l}\text { Active Focusing } \\
{[23] \text { SCNR, dB }}\end{array}$ & $\begin{array}{l}\text { Hybrid Active } \\
\text { Focusing } \\
\text { SCNR, dB }\end{array}$ \\
\hline 0.08 & $0^{*}$ & $0^{*}$ & 13.31 \\
0.16 & 3.16 & 6.06 & 21.73 \\
0.36 & 8.63 & 15.91 & 30.32 \\
0.72 & 18.72 & 22.24 & 33.14 \\
1.44 & 22.24 & 25.92 & 34.21 \\
\hline \hline
\end{tabular}




\section{List of figures}

Figure 1: $\quad$ Phase velocity dispersion curves for an 8inch schedule 40 (outer diameter: $219.1 \mathrm{~mm}$ and wall thickness: $8.18 \mathrm{~mm}$ ) steel pipe.

Figure 2: Displacement characteristics of the (a) $\mathrm{T}(0,1)$ and (b) $\mathrm{F}(1,2)$ wave modes.

Figure 3: $\quad$ Multimodal excitation (a) predicted displacement received from $1.5 \mathrm{~m}$ away from the point source excitation (b) Gated pulse (c) Gated-time reversed multimodal pulse used as an input to produce a focal spot.

Figure 4: $\quad$ Layout of the FE model to calculate focusing parameters.

Figure 5: Displacement magnitude results from the FE analysis at the time and axial position of focus (a) Unfocused (b) HAF and (c) polar plot for comparison of normalized displacement amplitude.

Figure 6: $\quad$ (a) 3D LDV experimental setup (b) area scan data collection (red line) (c) line scan data collection (dashed red line).

Figure 7: $\quad$ Experimental results (a) Isometric view of surface velocity from an unfocused excitation (b) Isometric view of surface velocity from the HAF excitation and (c) Polar plot comparing normalized amplitude from the FE analysis with experimental results at the focal point.

Figure 8: $\quad$ Schematics of the crack-like defects studied.

Figure 9: Illustration of SCNR calculation (using time-domain response from Defect ID: D-3). Defect range $D_{r}$ and coherent noise range $N_{r}$.

Figure 10: $\quad$ SCNR against crack circ.extent, $\lambda_{s}$ of active focusing, time reversal focusing and HAF.

Figure 11: Normalized defect amplitude against crack circ.extent, $\lambda_{s}$ of unfocused axisymmetric excitation, synthetic focusing and HAF. 
Figure 1: $\quad$ Phase velocity dispersion curves for an 8inch schedule 40 (outer diameter: $219.1 \mathrm{~mm}$ and wall thickness: $8.18 \mathrm{~mm}$ ) steel pipe.

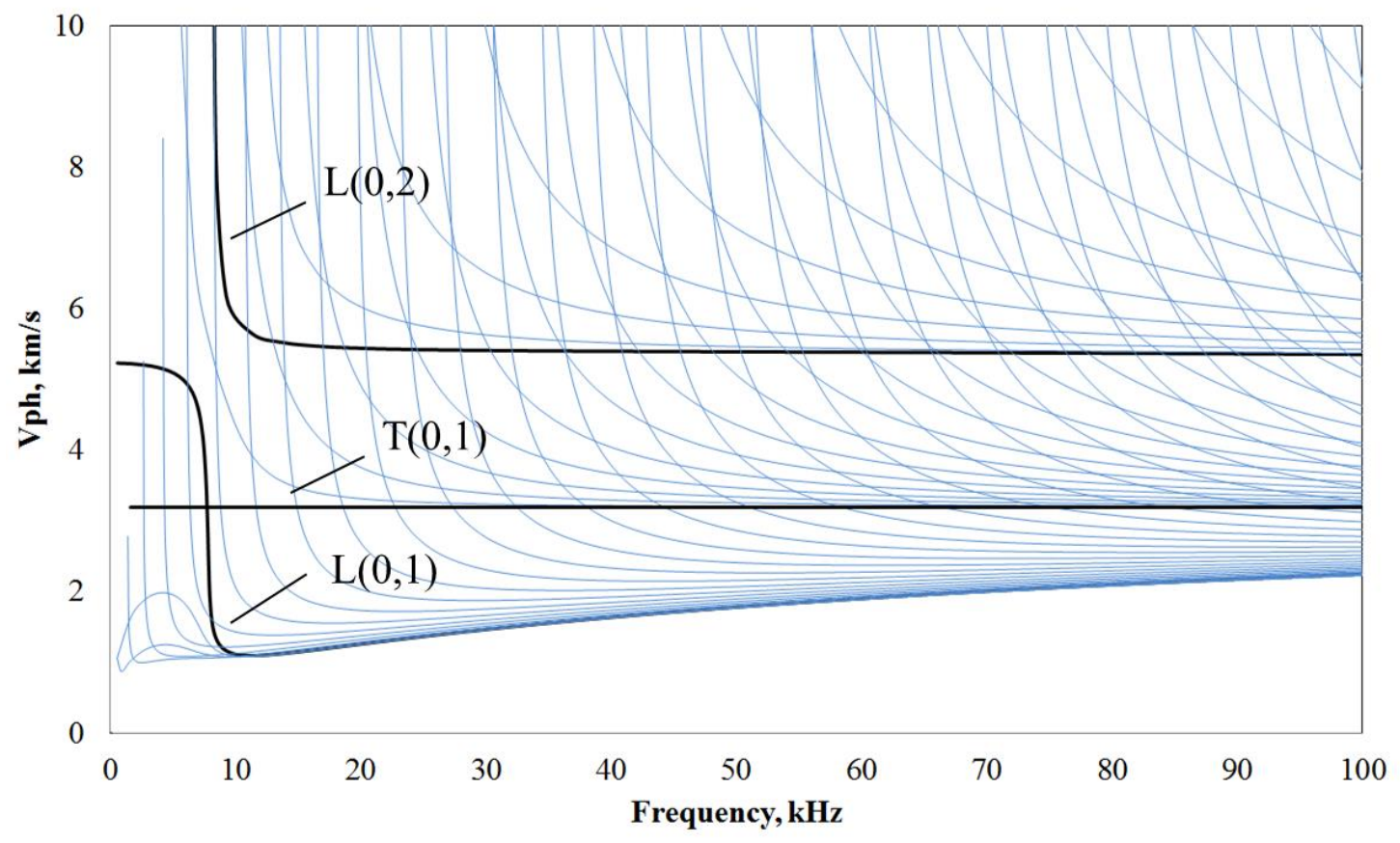


Figure 2: $\quad$ Displacement characteristics of the (a) $T(0,1)$ and (b) $F(1,2)$ wave modes.

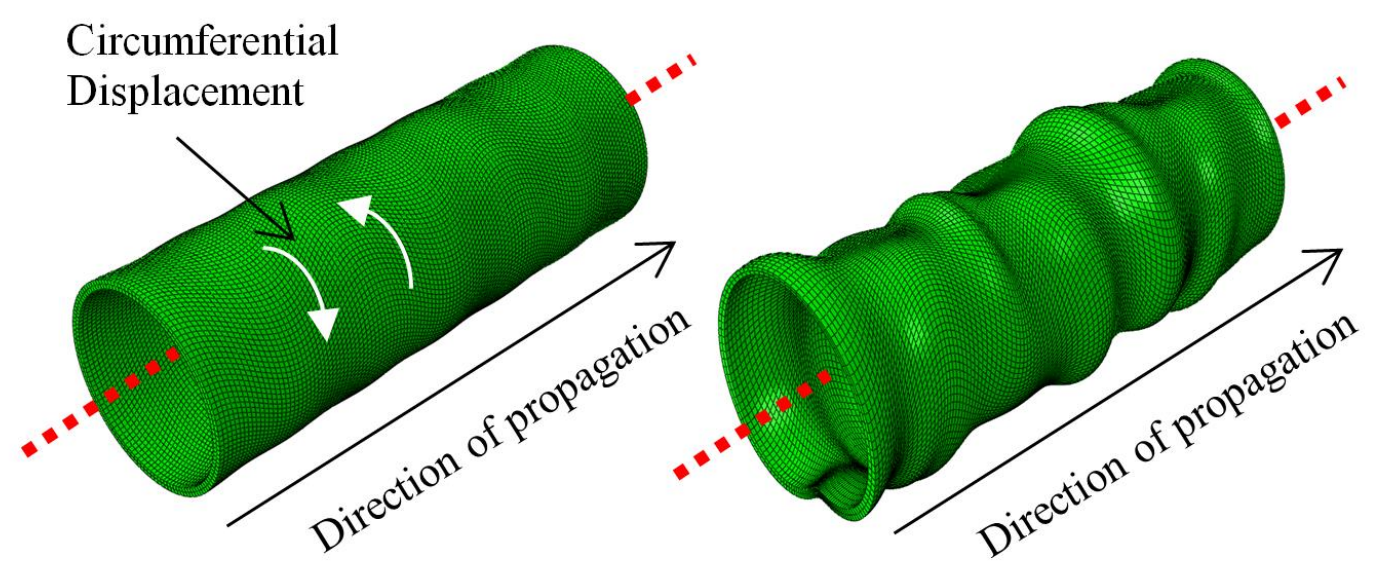


Figure 3: $\quad$ Multimodal excitation (a) predicted displacement received from $1.5 \mathrm{~m}$ away from the point source excitation (b) Gated pulse (c) Gated-time reversed multimodal pulse used as an input to produce a focal spot.

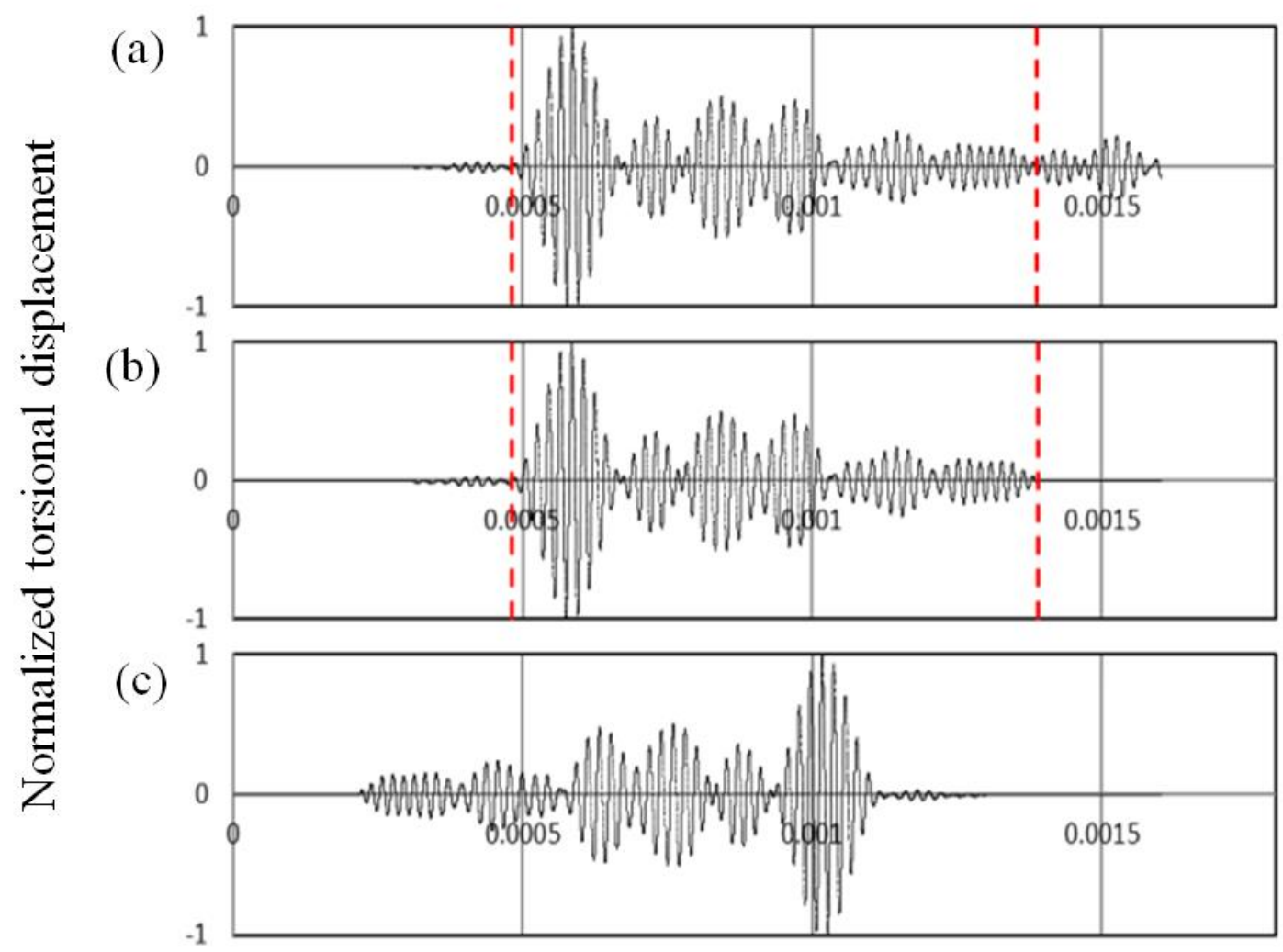

Time, s 
Figure 4: $\quad$ Layout of the FE model to calculate focusing parameters.

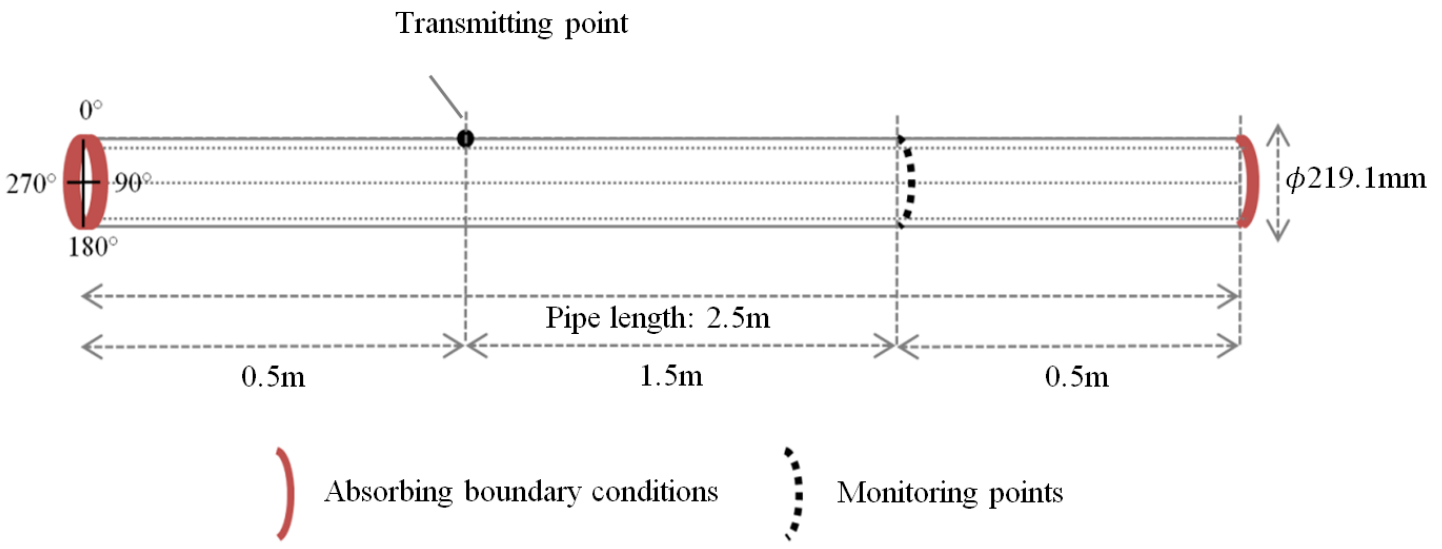


Figure 5: Displacement magnitude results from the FE analysis at the time and axial position of focus (a) Unfocused (b) HAF and (c) polar plot for comparison of normalized displacement amplitude.

(a)

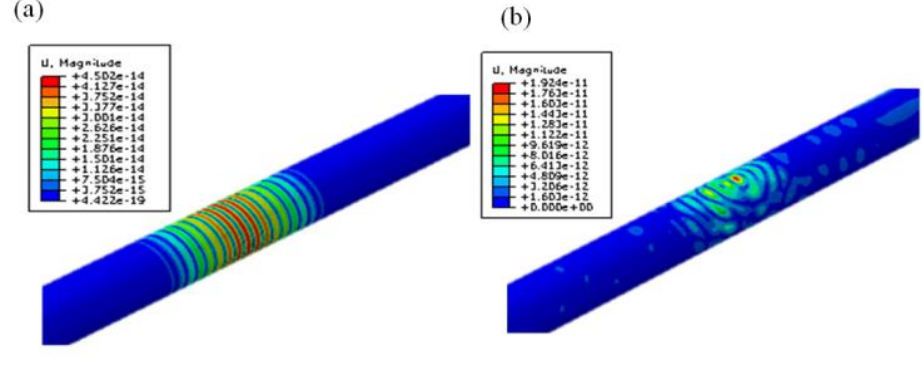

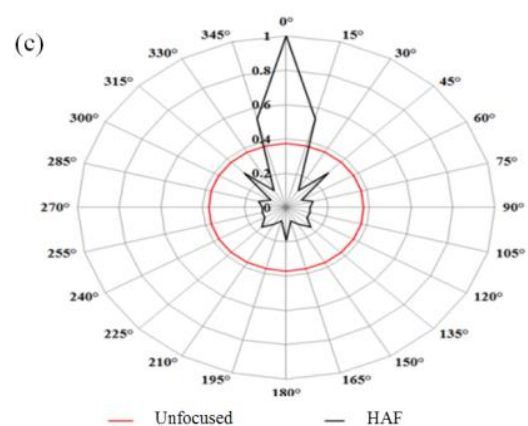


Figure 6: $\quad$ (a) 3D LDV experimental setup (b) area scan data collection (red line) (c) line scan data collection (dashed red line).

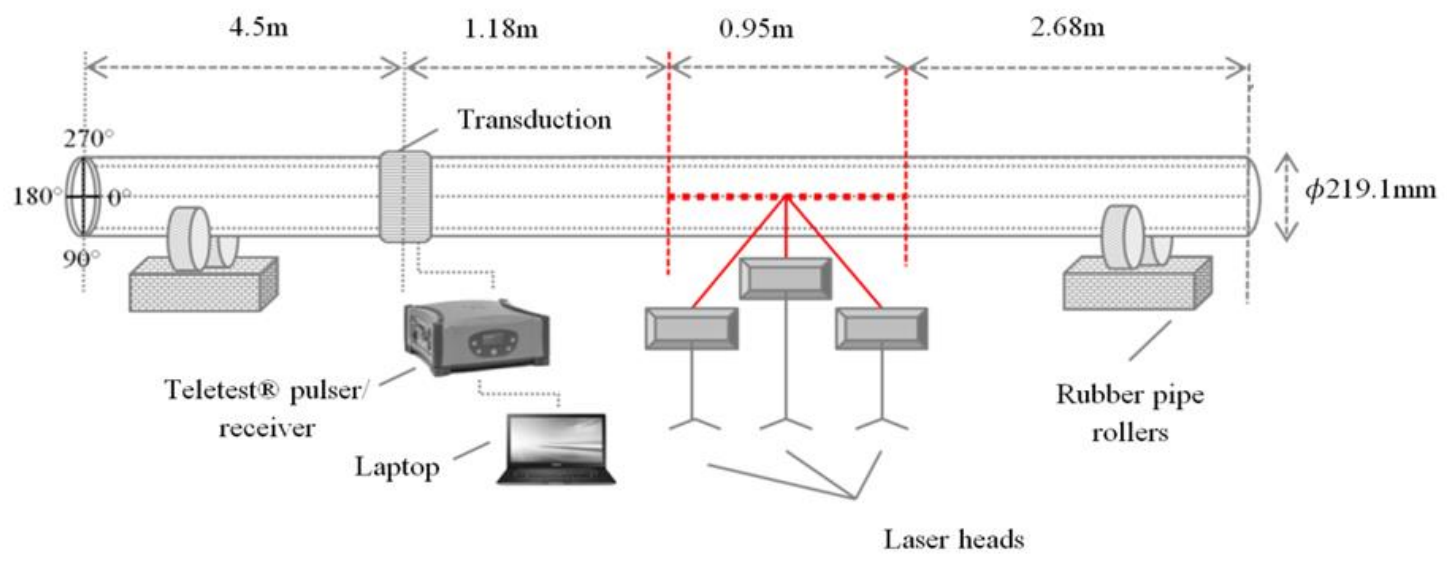

(a)

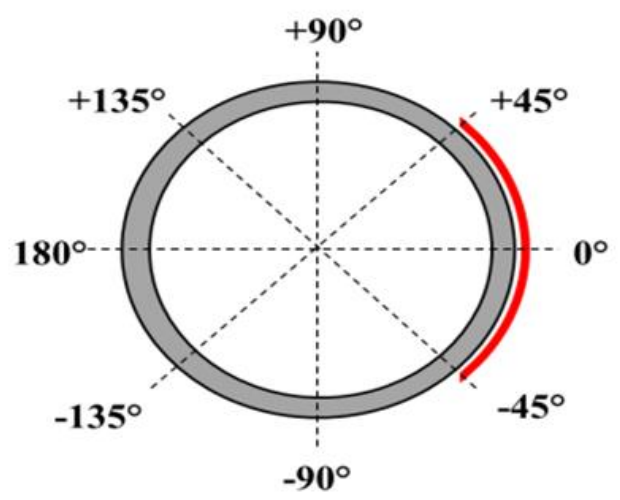

(b)

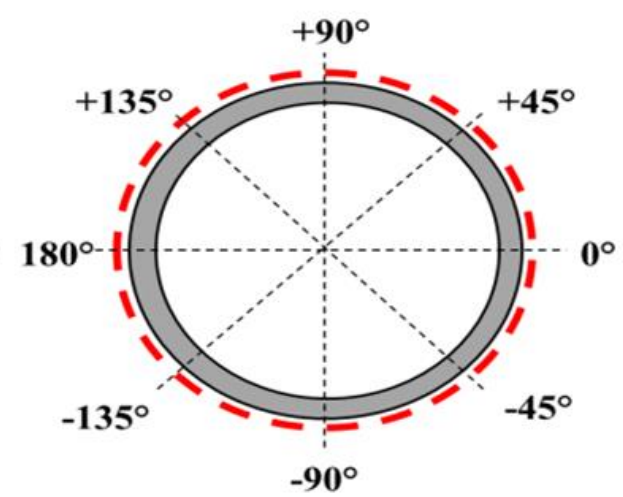

(c) 
Figure 7: $\quad$ Experimental results (a) Isometric view of surface velocity from an unfocused excitation (b) Isometric view of surface velocity from the HAF excitation and (c) Polar plot comparing normalized amplitude from the FE analysis with experimental results at the focal point.

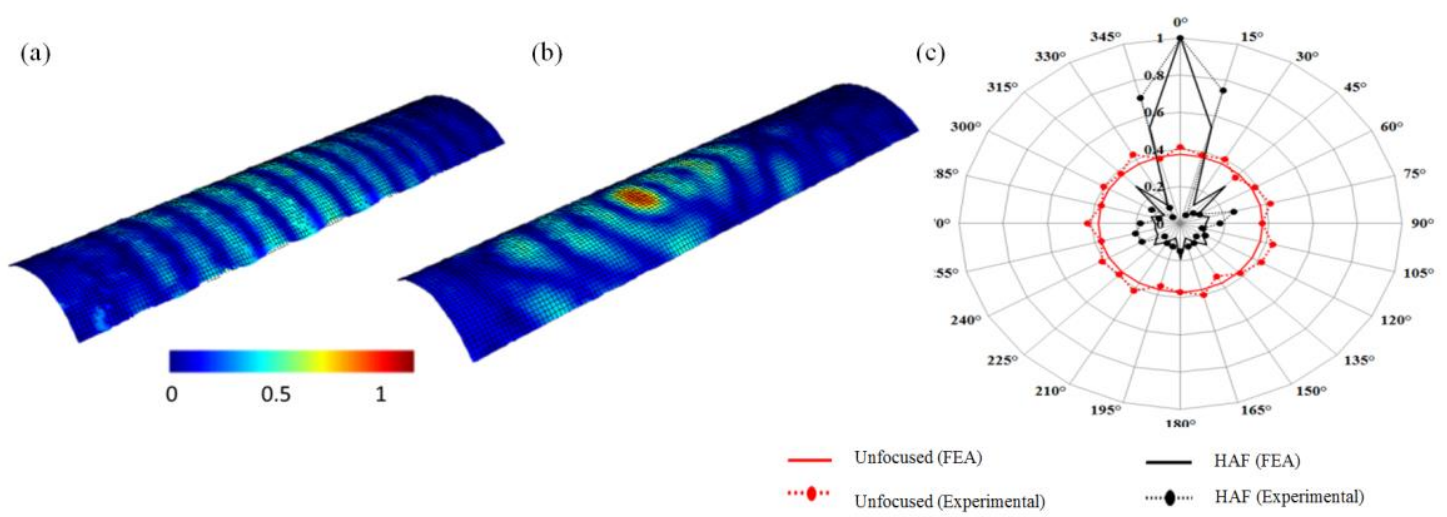


Figure 8: $\quad$ Schematics of the crack-like defects studied.
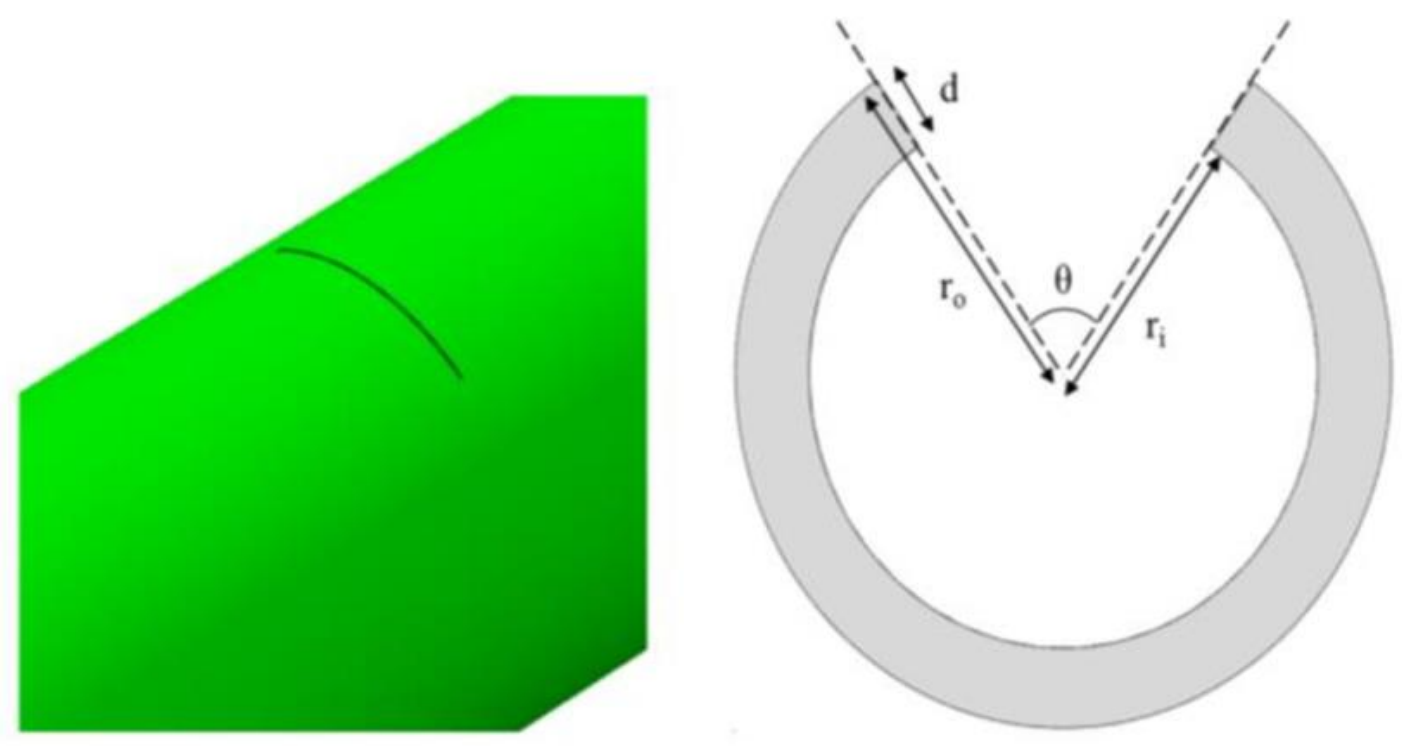
Figure 9: Illustration of SCNR calculation (using time-domain response from Defect ID: D-3). Defect range $D_{r}$ and coherent noise range $N_{r}$.

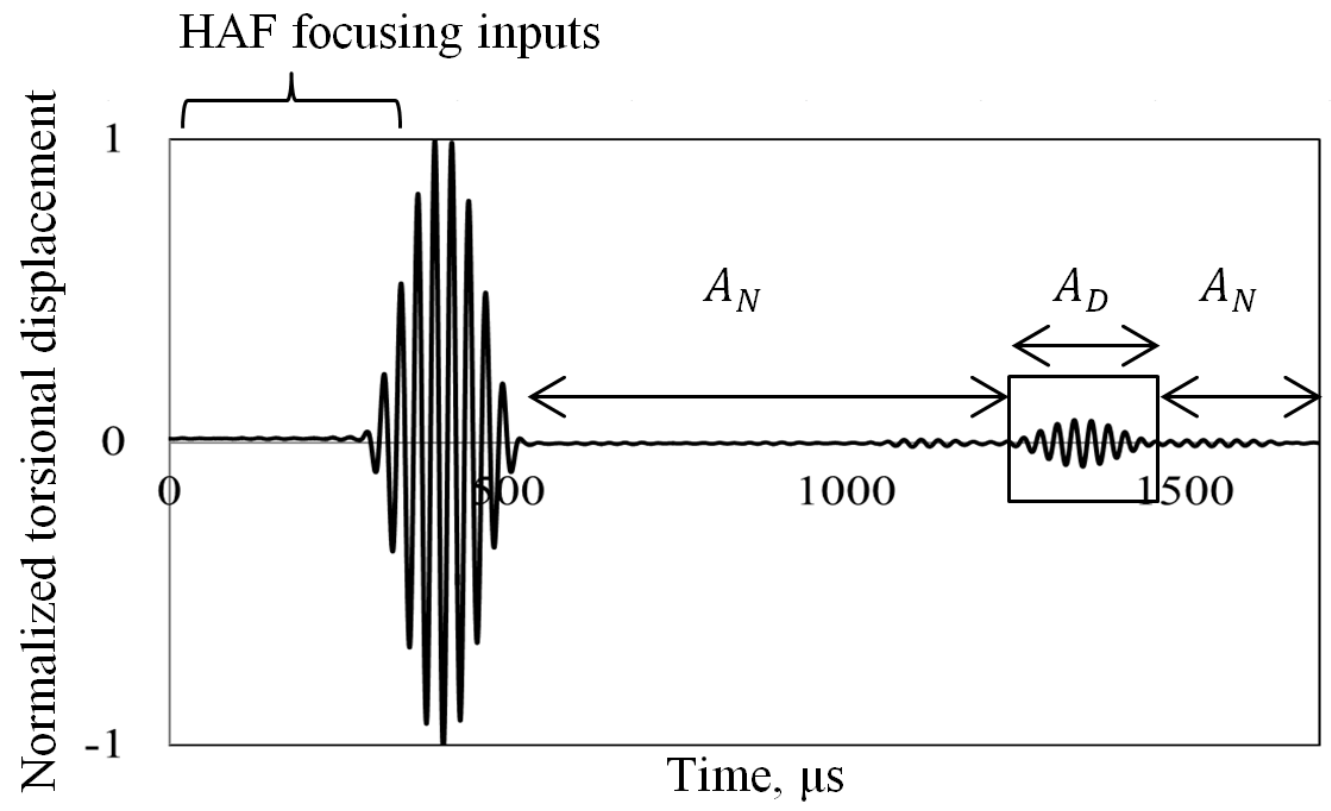


Figure 10: $\quad$ SCNR against crack circ.extent, $\lambda_{s}$ of active focusing, time reversal focusing and HAF.

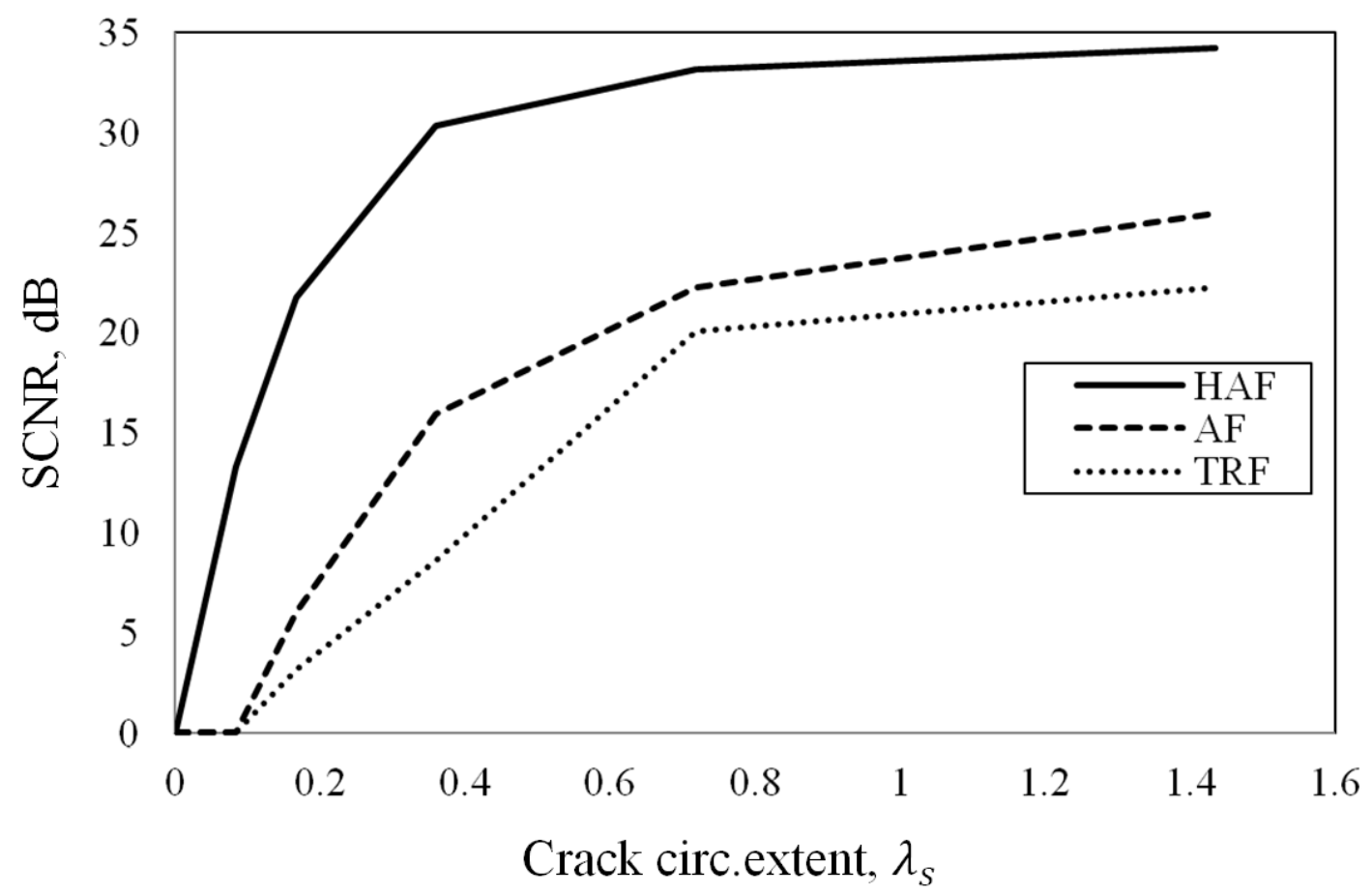


Figure 11: Normalized defect amplitude against crack circ.extent, $\lambda_{s}$ of unfocused axisymmetric excitation, synthetic focusing and HAF.

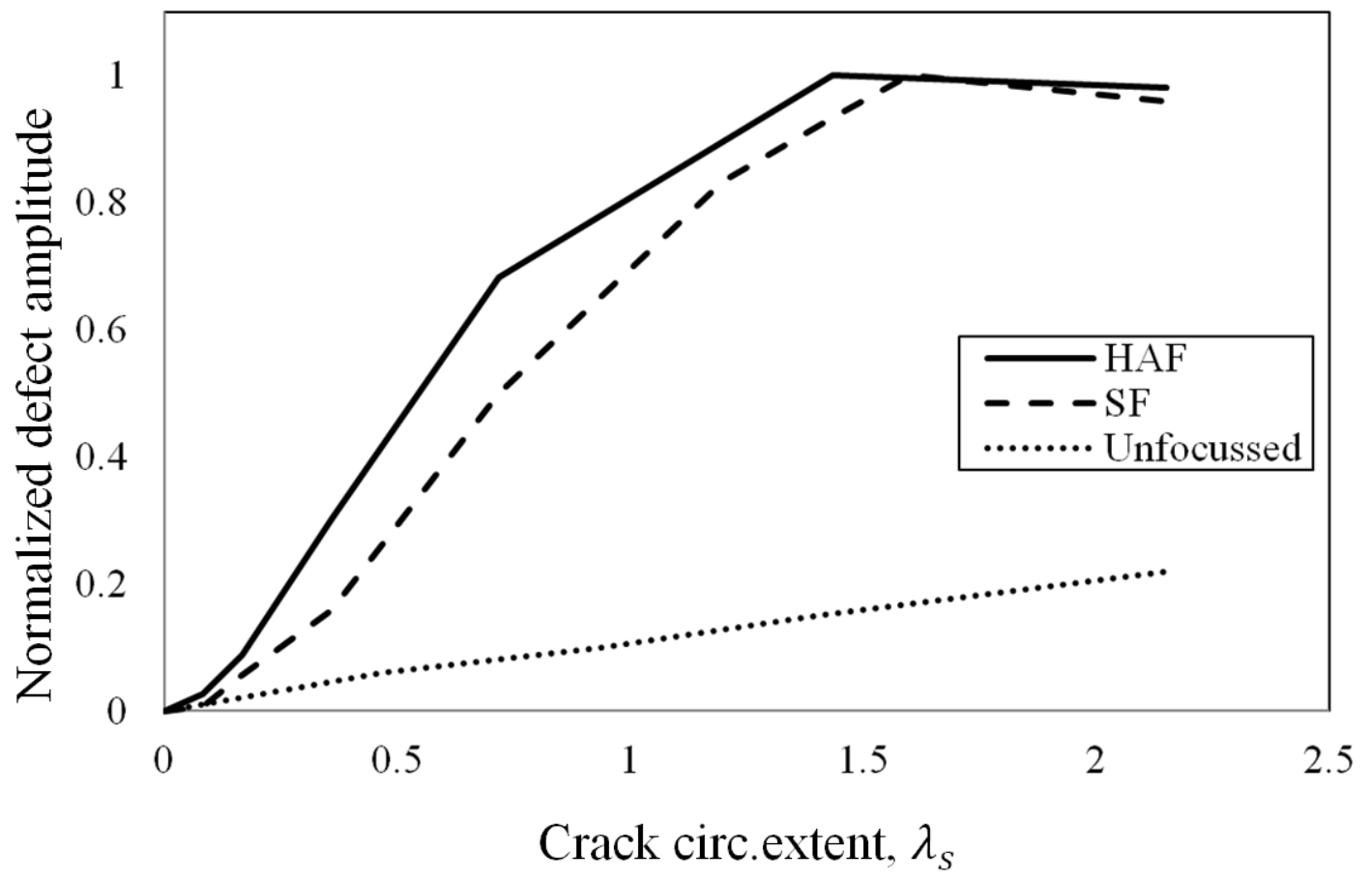

